\title{
Performance of Synthetic Aperture Compounding for in-vivo imaging
}

\author{
Hansen, Jens Munk; Jensen, Jørgen Arendt
}

Published in:

Proceedings of IEEE International Ultrasonics Symposium

Link to article, DOI:

10.1109/ULTSYM.2011.0282

Publication date:

2011

Document Version

Early version, also known as pre-print

Link back to DTU Orbit

Citation (APA):

Hansen, J. M., \& Jensen, J. A. (2011). Performance of Synthetic Aperture Compounding for in-vivo imaging. In Proceedings of IEEE International Ultrasonics Symposium (pp. 1148-1151). IEEE.

https://doi.org/10.1109/ULTSYM.2011.0282

\section{General rights}

Copyright and moral rights for the publications made accessible in the public portal are retained by the authors and/or other copyright owners and it is a condition of accessing publications that users recognise and abide by the legal requirements associated with these rights.

- Users may download and print one copy of any publication from the public portal for the purpose of private study or research.

- You may not further distribute the material or use it for any profit-making activity or commercial gain

- You may freely distribute the URL identifying the publication in the public portal

If you believe that this document breaches copyright please contact us providing details, and we will remove access to the work immediately and investigate your claim. 
Paper presented at the IEEE International Ultrasonics Symposium, Orlando, Florida, 2011:

\section{Performance of Synthetic Aperture Compounding for in-vivo imaging}

Jens Munk Hansen and Jørgen Arendt Jensen

Center for Fast Ultrasound Imaging,

Biomedical Engineering group, Department of Electrical Engineering, Bldg. 349,

Technical University of Denmark, DK-2800 Kgs. Lyngby, Denmark

To be published in Proceedings of IEEE International Ultrasonics Symposium, Orlando, Florida, 2011. 


\title{
Performance of Synthetic Aperture Compounding for in-vivo imaging
}

\author{
Jens Munk Hansen and Jørgen Arendt Jensen \\ Center for Fast Ultrasound Imaging, Dept. of Elec. Eng. Bldg. 349, \\ Technical University of Denmark, DK-2800 Kgs. Lyngby, Denmark
}

\begin{abstract}
A method for synthetic aperture compounding (SAC) is applied to data from water tank measurements, data from a tissue-mimicking phantom, and clinical data from the abdomen of a healthy 27 year old male. Further, using this method compounding can be obtained without any loss in temporal resolution. The water tank measurements reveal an improved detail resolution of $45 \%$ when comparing SAC to conventional compounding and an improvement of $22 \%$, when comparing to synthetic aperture (SA) imaging. The cystic resolution at $12 \mathrm{~dB}$ is improved by $50 \%$ and $12 \%$ when comparing SAC to conventional compounding and $\mathrm{SA}$ imaging respectively. The tissue phantom measurements show a $3.2 \mathrm{~dB}$ improvement of the normalized information density (NID) when comparing images formed using SAC to conventional compound images and an improvement of 2 dB for a comparison between SAC imaging and SA imaging. For the clinical images, contrast ratios (CR) are computed between regions in the portal and hepatic veins and the surrounding tissue. An average improvement of $15 \%$ is obtained when comparing SAC images to SA images without compounding.
\end{abstract}

\section{INTRODUCTION}

Medical ultrasound imaging is used for many purposes, e.g. for localizing and classifying cysts, lesions, and other processes. Almost any mass is first observed using B-mode imaging and later classified using e.g. color flow, strain, or biopsies. It is therefore important that the B-mode images have high contrast. Like all imaging modalities, ultrasound is subject to a number of inherent artifacts that compromise image quality. The most prominent artifact is the degradation by coherent wave interference, known as 'speckle', which gives a granular appearance to an otherwise homogeneous region of parenchyma [1], [2]. The speckle reduces image contrast and diminishes the possibilities for detection of lowcontrast regions.

A successful approach to remedy the speckle artifacts is spatial compounding, where images are acquired from a number of directions and combined after envelope-detection [3], [4]. Today, spatial compounding is implemented in all state of the art, high-end ultrasound systems and available when using a low pitch transducer with a fairly high number of independent channels. A drawback of conventional compounding is either a reduction of the frame rate or a reduction of the true temporal resolution. This paper investigates an approach based on synthetic aperture imaging, where compounding can be obtained without any loss in temporal resolution.

The organization of this paper is as follows: First, some performance measures for ultrasound imaging systems are defined. Next, spatial compounding is described as used for images formed using dynamic receive focusing (DRF), which we will refer to as DRFC. After a brief introduction to synthetic aperture (SA) imaging, the synthetic aperture compounding (SAC) is described in full detail. In the results section, performance measures are extracted from water tank and phantom measurements and using a similar setup, the first clinical image obtained using SAC is presented. Finally, a discussion of the results and possibilities for improvements.

\section{Performance Measures}

Development of new ultrasound imaging methods includes several quality and performance assessment stages. To assess the image quality both the detail and contrast resolution are studied. They can both be measured and quantized through a combination of water tank and phantom measurements, but often extensive clinical evaluations are also carried out to ensure that the desired clinical performance is met. In this paper, the main focus will be on the following measures, which can be obtained from water tank and phantom measurements alone.

a) Detail resolution: The detail resolution is the separation at which identically point targets can be distinguished. It is distinguished by the main lobe width of the point-spreadfunction (PSF) and the $6 \mathrm{~dB}$ axial pulse length, i.e. the lateral and axial full width at half maximum (FWHM) of the PSF.

b) Cystic resolution: The detail resolution alone is not appropriate for comparing the imaging performance of medical ultrasound systems, since it ignores what is outside the main lobe, which have a significant impact on the image of wide dynamic range systems. Acoustic clutter from surrounding objects fill-in images of anechoic objects such as cysts, or weakly echogenic objects such as blood vessels, and reduces their detectability. The ability to detect anechoic or weakly echogenic objects in the presence of strong surrounding objects is sometimes referred to as contrast resolution and was first introduced as "cystic resolution" [5]. The contrast resolution can be quantized by the clutter energy to total energy ratio (CTR). The CTR is defined as the ratio of the PSF energy outside a circular region with radius $R$ to the total PSF energy. For a large ensemble of measurements, the CTR is also a measure of the difference between the average level of a cyst's center and the background. To get a single measure, one either measures the drop in brightness for a fixed radius $R$ or the radius of a cyst, which can be observed at a fixed level, e.g. $R_{12 \mathrm{~dB}}$. 
c) Tissue contrast resolution: Most commonly, contrast resolution refers to the ability to distinguish echogenicity differences between a region of interest (ROI) and the background, tissue contrast resolution. Tissue contrast resolution is often quantified by either contrast ratio (CR) or contrast-tonoise ratio (CNR), which are both object dependent and where latter is improved on increasing object contrast as well as by lowering the variance.

d) Normalized information density: The primary source of contrast resolution loss is coherent wave interference also known as speckle. This artifact is reflected in the signal-tonoise ratio (SNR) at a point defined as the expectation value of the magnitude of the received signal, $\mu_{V}=\mathbb{E}(V)$ and in units of its standard deviation, $\sigma_{V}$. In the literature, this is referred to as the signal-to-noise ratio at a point $\mathrm{SNR}_{0}$, and if the envelope-detected signals, $V$ follow Rayleigh statistics, then for a fully developed speckle, the theoretical value of $\mathrm{SNR}_{0}$ is 1.91 [2]. This value is increased by spatial compounding [6] and an increase is associated with improved image contrast. $\mathrm{SNR}_{0}$ is object independent, but theoretically it can be shown that the SNR for a difference signal scales with the square root of the number of independent speckle cells [7], so somehow it would be beneficial to incorporate speckle size. Such a measure was introduced by Ustuner et. al. referred to as normalized information density (NID), which reflects the ability of a system to distinguish $1 \mathrm{~dB}$ brightness differences in the presence of fully-developed speckle [8]

$$
\mathrm{NID}=\frac{\mathrm{SNR}_{0}^{2}}{2 S}=\frac{\left(\mu_{V} / \sigma_{V}\right)^{2}}{2 S}
$$

where $S$ is the average speckle size, which can be obtained from the auto-covariance function of the intensity.

\section{METHODS}

\section{A. Compounding}

For conventional spatial compounding, a low-pitch linear or convex array is used. A subset of the elements is selected as the current active aperture, and this aperture is used as a phased array to steer the beam in a direction making an angle $\theta$ with a normal to the transducer surface. The active aperture is then moved, until scan lines originating from the entire extent of the array are recorded. This procedure is repeated for angles $\theta_{i}, i=1, \ldots N_{\theta}$, where $N_{\theta}$ is the number of angles to be used for compounding. Next, the $N_{\theta}$ sub-images are envelope-detected and added using scan-line conversion. The resulting image has a reduced speckle appearance, a lower noise floor, grating lobes (if present) are lowered, and boundaries subject to non-normal incidence are more visible, and image shadowing is confined to a smaller triangular region behind the attenuating masses or boundaries. The region where all sub-images overlap is referred to as the fully-compounded region.

A drawback of conventional compounding is either a reduction of the frame rate or a reduction of the true temporal resolution. In the case of the latter, the temporal resolution is reduced due to images being buffered to achieve an apparent high frame rate.

\section{B. Synthetic Aperture}

A typical setup for synthesizing both the transmit and receive aperture is to acquire data by emitting a spherical wave with a subaperture and receiving with all of the elements [9]. This procedure is then repeated for all emissions and a complete data set is acquired. Using this data set, any beam and focusing can be synthesized.

For transmit focusing, delays and apodizations are calculated for each point in the image to construct signals, which at each point sum up coherently. For receive focusing, another set of delays and apodizations are applied to the signals received from the individual transducer elements and then a weighted sum is performed. In addition, the apodization can be adjusted to even out the resolution over a range of depths to maintain a constant resolution by realizing a fixed F-number using an expanding or contracting aperture for transmit and receive beamformation, respectively. A major advantage of synthetic aperture imaging is the possibility to focus everywhere in the image resulting in a better resolution. Further, this can be achieved using the same amount of time for data acquisition as for conventional imaging. The price for the resolution though, is a huge increase in RF data processing - an increase by the number of lines to beamform for each pulse emission.

\section{Synthetic Aperture Compounding}

To perform compounding using synthetic aperture data, apodizations are calculated for each image point corresponding to imaging the point using a focused beam centered around the point and making an angle $\theta$ with a normal to the transducer surface. In Fig. 1, it is shown for a single point, how the transmit apodization for an emission with transmit origin $\vec{x}_{\text {origin }}$ are calculated using the distance from the transmit origin $\vec{x}_{\mathrm{xmt}}$ to the "scan-line". Similarly, receive apodizations

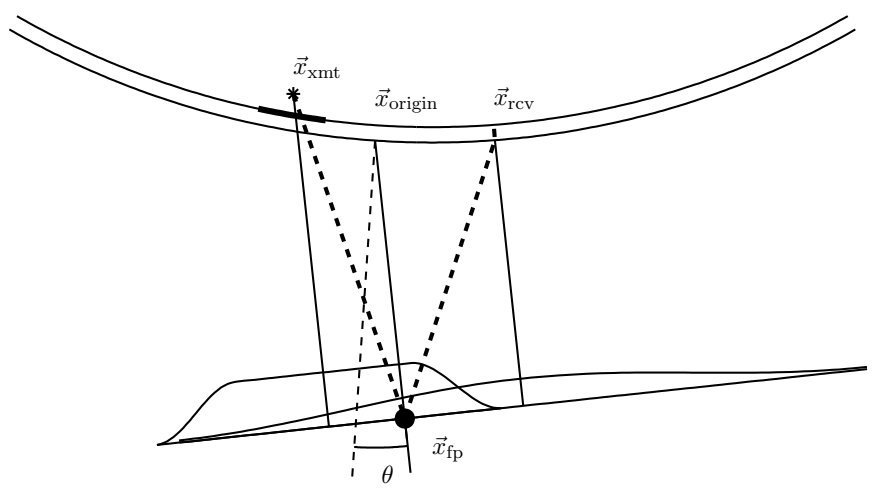

Figure 1. Wave propagation path (thick dashed line) for calculating time-offlight for synthetic aperture focusing for an emission with origin $\vec{x}_{\text {origin }}$ and data acquired with the element positioned at $\vec{x}_{\mathrm{rcv}}$. The transmit and receive apodizations can be read-off the figure as the intersections of the scan line and the Tukey and Hamming profile respectively.

are calculated for each point and applied to the signals received 
from the individual receiving elements of each transmission. The receive apodization values are likewise computed using the distance from the "scan-line" to now the position of the receiving elements. The delay calculation are identical to what is used for synthetic aperture imaging without compounding. The RF data are beamformed as in-phase and quadrature components, and the envelope computed as the absolute value. This rather complex procedure is repeated for angles $\theta_{i}$, $i=1, \ldots N_{\theta}$, where $N_{\theta}$ is the number of angles to be used for compounding. Finally, the compound image are constructed by addition or multiplication of the enveloped images. An implementation of this method uses a buffer for $N_{\theta}$ images and calculates the delays for each pixel only once. In this way, a compound image is ready immediately after the last emission and only the processing resources limits the number of angles used.

\section{RESULTS}

To investigate the contrast and detail resolution for SAC a setup using a commercial $3 \mathrm{MHz}, 192$ element, $\lambda$ pitch convex array (BK Medical) was used for water tank and tissue phantom measurements. The sampling was done using an experimental ultrasound scanning system, SARUS, capable of storing 12-bit individual channel data at $70 \mathrm{MHz}$ [10]. For SA and SAC imaging, a 16 element subaperture was used for 192 unfocused emissions. For each emission, data from all 192 channels were stored. For comparison, for DRF and DRFC, a 64 element subaperture was used for 192 focused emissions for each compound angle. For each emission, data from all 192 channels were stored. For all emissions, a two-cycle sinusoid was used for excitation together with a Hamming apodization on the active subaperture. Compounding for DRF and SA data was done using 5 angles with an angular separation of 5 degrees.

For processing, a small parameter study was made to find the optimal F\#'s for dynamic apodization to achieve the best $R_{12 \mathrm{~dB}}$ for wires at depths $15,40,65$, and $90 \mathrm{~mm}$.

\section{A. Water phantom}

In Table I, the detail resolution and contrast resolution (for anechoic objects) is measured for the wire at depth $65 \mathrm{~mm}$, shown in Fig. 2 using the four imaging techniques. For both

Table I

\begin{tabular}{lcccc}
\hline & $R_{12 \mathrm{~dB}}$ & $\mathrm{FWHM}_{\text {lat }}$ & $\mathrm{FWHM}_{\mathrm{ax}}[\mathrm{mm}]$ & Area $_{6 \mathrm{~dB}}\left[\mathrm{~mm}^{2}\right]$ \\
\hline DRF & 1.25 & 1.41 & 0.70 & 3.08 \\
DRFC & 1.19 & 1.37 & 0.70 & 3.00 \\
SA & 0.68 & 1.13 & 0.59 & 2.10 \\
SAC & 0.60 & 0.83 & 0.63 & 1.65 \\
\hline
\end{tabular}

the DRF and SA, compounding results in an improved lateral resolution, but only for SA this comes at the cost of a worse axial resolution. The cystic resolution, $R_{12 \mathrm{~dB}}$ is also improved.

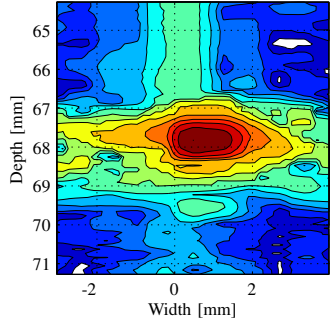

(a) DRF

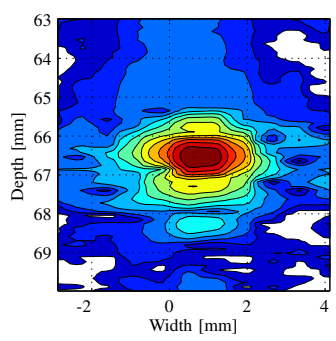

(c) SA

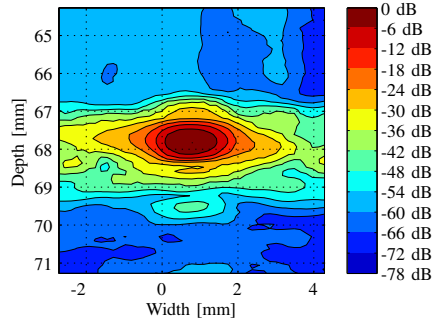

(b) DRFC

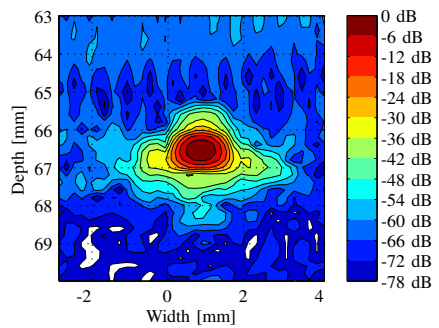

(d) SAC
Figure 2. PSF images obtained using water tank phantom with wires.

Finally, we note that the CTR as well as the clutter ratio is much improved for both SA and SAC compared to DRF and DRFC.

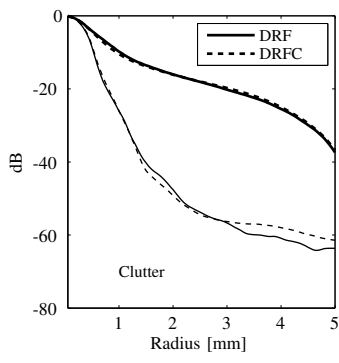

(a) DRF and DRFC

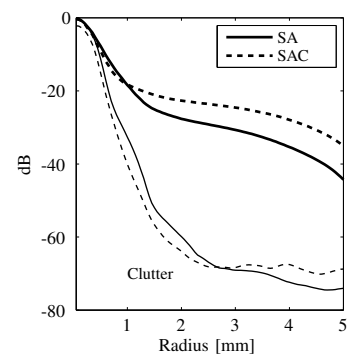

(b) SA and SAC
Figure 3. CTR for DRF and SA (thick dashed lines) and for DRFC and SAC (thick solid lines). Clutter levels are shown below as thin dashed and solid lines for the wire at $65 \mathrm{~mm}$, for images with and without compounding respectively.

\section{B. Tissue phantom}

To obtain measures for tissue contrast resolution, a tissue mimicking phantom was scanned using the same configurations. In Fig. 4, the resulting images are presented and in Table II, $\mathrm{SNR}_{0}$, speckle size, and NID measures are presented computed as an average inside the regions shown in Fig. 4. A small increase in $\mathrm{SNR}_{0}$ followed by an increase in speckle size is seen for DRFC, when compared to DRF. This is much less than anticipated and deserves future investigation. Consequently, only a $1 \%$ increase in NID is observed. For SA, no increase in $\mathrm{SNR}_{0}$ is observed, a large decrease in speckle size is observed, and the NID is improved dramatically. 


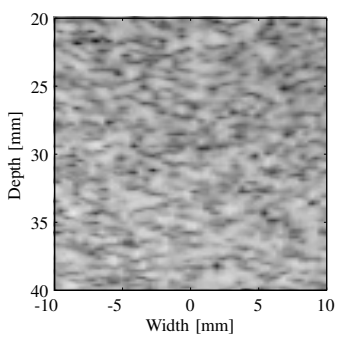

(a) DRF

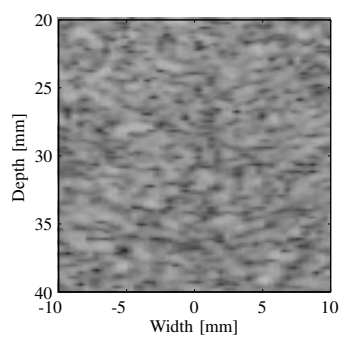

(c) $\mathrm{SA}$

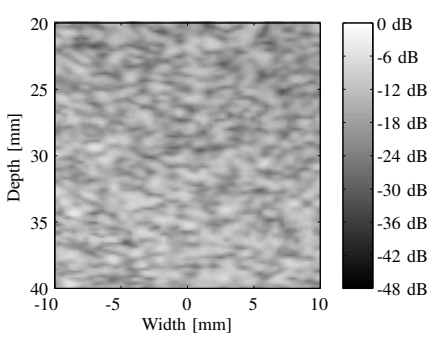

(b) DRFC

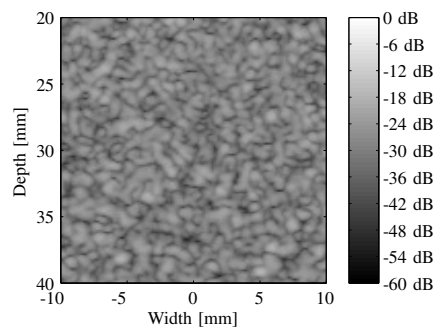

(d) SAC

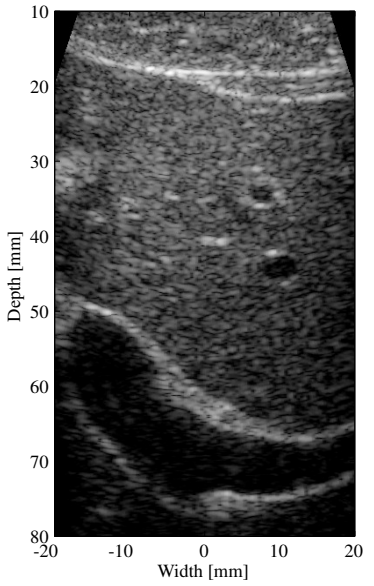

(a) SA

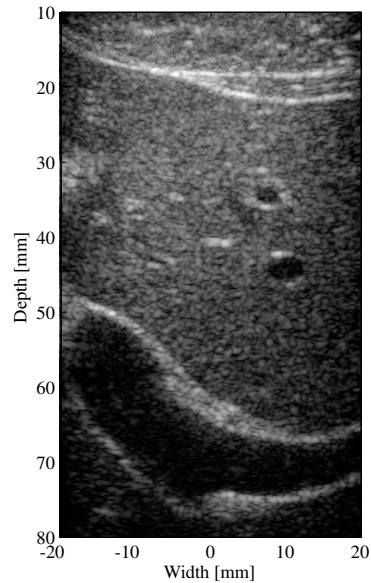

(b) SAC
Figure 5. The images (a) and (b) show the abdomen of a healthy 27 year old male with and without compounding. Dynamic range is $60 \mathrm{~dB}$.

Table III

Figure 4. Speckle images obtained using a tissue-mimicking phantom.

Table II

\begin{tabular}{lccc}
\hline & $\mathrm{SNR}_{0}$ & Speckle size $\left[\mathrm{mm}^{2}\right]$ & NID $\left[\mathrm{dBmm}^{-2}\right]$ \\
\hline DRF & 1.88 & 0.66 & 4.29 \\
DRFC & 1.96 & 0.73 & 4.23 \\
SA & 2.01 & 0.57 & 5.50 \\
SAC & 1.95 & 0.34 & 7.45 \\
\hline
\end{tabular}

\section{Clinical images}

The measurements for the clinical images were performed using an experimental ultrasound scanning system, RASMUS, capable of storing 12-bit individual channel data at $40 \mathrm{MHz}$. For imaging, an 11 element subaperture is used for each emission using a $20 \mu \mathrm{s}$ FM signal. For receiving 128 channels are sampled. The transducer used is a commercial $5.5 \mathrm{MHz}$, 128 element convex array with $\lambda$ pitch (BK Medical) [11].

The data were processed exactly like for the water and tissue phantoms taking into account the different excitation. In Fig. 5, the first clinical image obtained using SAC is presented. The portal vein branch is in the center of the image and the hepatic veins are located to the left above. CR and CNR values are computed between regions in the portal and hepatic veins and the surrounding tissue. The resulting values are presented in Table III.

\section{CONClusion}

The water tank and tissue-mimicking phantom measurements show an improved lateral resolution and an improved NID for the suggested method for compounding using synthetic aperture data. An improved contrast resolution is also observed for the clinical data and it is definitely worth continuing studying this method for further evidence of its work

\begin{tabular}{|c|c|c|c|}
\hline & SA & $\mathrm{SAC}$ & \\
\hline $\mathrm{CR}(\mathrm{dB})$ & -12.59 & -13.07 & స్తుర \\
\hline CNR & -1.34 & -1.30 & $\bar{\varnothing}$ \\
\hline $\mathrm{CR}(\mathrm{dB})$ & -16.77 & -18.41 & . \\
\hline CNR & -2.66 & -2.88 & $\underline{2}$ \\
\hline
\end{tabular}

\section{REFERENCES}

[1] C. Burckhardt, "Speckle in ultrasound B-mode scans," IEEE Trans. Son. Ultrason., vol. SU-25, no. 1, pp. 1-6, Jan. 1978.

[2] R. F. Wagner, S. W. Smith, J. M. Sandrick, and H. Lopez, "Statistics of speckle in ultrasound B-scans," IEEE Trans. Son. Ultrason., vol. 30, pp. 156-163, 1983.

[3] D. Shattuck and O. von Ramm, "Compounding scanning with a phased array," Ultrason. Imaging, vol. 4, pp. 93-107, 1982.

[4] G. E. Trahey, S. W. Smith, and O. T. von Ramm, "Speckle pattern correlation with lateral aperture translation: Experimental results and implications for spatial compounding," IEEE Trans. Ultrason., Ferroelec., Freq. Contr., vol. UFFC-33, pp. 257-264, 1986.

[5] D. Vilkomerson, J. Greenleaf, and V. Dutt, "Towards a Resolution Metric for Medical Ultrasound Imaging," in Proc. IEEE Ultrason. Symp., 1995, pp. 1405-1410.

[6] S. K. Jespersen, J. E. Wilhjelm, and H. Sillesen, "Multi-angle compound imaging," Ultrason. Imaging, vol. 20, pp. 81-102, 1998.

[7] S. W. Smith, R. F. Wagner, J. M. Sandrik, and H. Lopez, "Low contrast detectability and contrast/detail analysis in medical ultrasound," IEEE Trans. Son. Ultrason., vol. 30, pp. 164-173, 1983.

[8] K. F. Ustuner and G. L. Holley, "Ultrasound imaging system performance assessment," presented at 2003 AAPM Annual Meeting, 2003.

[9] G. R. Lockwood, J. R. Talman, and S. S. Brunke, "Real-time 3-D ultrasound imaging using sparse synthetic aperture beamforming," IEEE Trans. Ultrason., Ferroelec., Freq. Contr., vol. 45, pp. 980-988, 1998.

[10] J. A. Jensen, S. Nikolov, B. Tomov, F. Gran, M. Hansen, and T. V. Hansen, "Specification of SARUS: the synthetic aperture real-time ultrasound scanner," Ørsted•DTU, Technical University of Denmark, Tech. Rep., 2006.

[11] K. L. Gammelmark and J. A. Jensen, "Experimental study of convex coded synthetic transmit aperture imaging," in Proc. IEEE Ultrason. Symp., 2002, pp. 1573-1576. 